\title{
Misfit effects in adhesion calculations
}

\author{
Jurgen Schnitker and David J Srolovitz \\ Department of Materials Science and Engineering, University of Michigan, Ann Arbor, \\ MI 48109-2136, USA
}

Received 7 December 1997, accepted for publication 22 December 1997

\begin{abstract}
The work of adhesion of bimaterial interfaces is commonly computed using quantum mechanical methods in which the two materials are strained into coherency. There is no relaxation of the coherency by the formation of an array of interfacial misfit dislocations, contrary to what is commonly observed for essentially all systems other than very thin films. In this paper, we investigate the errors introduced into the work of adhesion associated with the assumption of coherency. Series of atomistic simulations in two and three dimensions are performed using a simple Lennard-Jones-type model potential. We demonstrate that the assumption of coherency introduces errors that increase rapidly with misfit (for small misfit) and can easily be of the order of several tens of percent. We trace the source of these errors to the neglect of the elastic fields of misfit dislocations and to the variation in the number of bonds per unit interfacial area with misfit when coherency is assumed. Suggestions are made to minimize and/or correct for this error.
\end{abstract}

\section{Introduction}

The work of adhesion or adhesive energy is an essential quantity for the rational design of multiphase materials [1]. It is also relevant for many other engineering applications that involve heterophase interfaces, such as microelectronics and catalyst supports [2]. Unfortunately, adhesion energies are notoriously difficult to measure, and first-principles calculations-provided they can be implemented-often provide the most reliable data. Similar issues arise in the determination of surface energies.

In this paper, we address one particular problem that always has to be faced in firstprinciples calculations involving interfaces between bulk samples of two different materials [3-13]. We will address the effects of the lattice misfit (i.e. mismatch in lattice parameters and/or crystal symmetry) on interfacial adhesion. There are several ways in which this misfit can be manifested at the interface. In one extreme, the atoms of both lattices are at (or very near to) their respective bulk equilibrium positions, thus giving rise to completely incoherent interfaces (at least in the absence of interfacial relaxation). In the other extreme, one of the lattices is strained so as to lead to complete coherency-a situation that in practice can only be realized for very thin films, because the strain energy increases in direct proportion to the film thickness. In a third scenario, areas of local coherency alternate with misfit dislocations that spatially localize the differences in lattice parameters between the two materials, thus giving rise to commonly occurring 'semicoherent' interfaces [13]. It is the effects of this misfit relaxation on the adhesion energy which is the focus of this study.

An essential element in the computer modelling of condensed phases is the use of periodic boundary conditions. The specific problem of adhesion calculations is that the periodic boundary conditions have to be satisfied simultaneously for two lattices with two 
different lattice parameters. For example, for a lattice mismatch of $10 \%$ a supercell has to be used that contains 11 unit cells of the smaller component for every 10 unit cells of the larger component in the directions parallel to the interface. A first complication arises in that such combinations of different numbers of unit cells to accommodate the misfit are possible only when the misfit strain is a rational number. If the misfit strain is irrational, it can be accommodated to high precision using very large supercells. An even bigger problem, however, is that the minimum size of the supercell needed to accommodate this misfit gets larger as the misfit decreases. Unfortunately, the use of very large supercells is not an option for a first-principles quantum-mechanical calculation, mainly because of the well known rapid increase of the computational cost with system size. Therefore, such calculations are usually implemented using a small supercell and one unique lattice parameter for both of the materials that are in contact; i.e. straining the lattices into perfect coherency. Hence misfit dislocations are artificially omitted.

Little is known about the ramifications of this geometric constraint (used in most adhesion calculations) for the adhesive energy. The purpose of this study is to provide insight into the consequences of employing this constraint. The idea is to replace the quantum mechanical description by a simple model system that can be analysed in detail for a variety of boundary conditions. The model systems used consist of either two-dimensional (2D) or three-dimensional (3D) close-packed crystals meeting at a heterophase interface, with all interactions described by simple Lennard-Jones-type potentials. We emphasize the pure model character of this system description. Although there have been instances where Lennard-Jones potentials have been used to study misfit dislocations between metals [14] and other adhesion-related phenomena [15], it has to be stressed that a realistic description of any specific, 'real' materials is not the goal of the present study.

To further reduce the complexity of the model description, we choose as many physical parameters as possible to be the same for the two crystals. Only the particle sizes of the two species differ so as to have a systematic variation of the lattice mismatch as the fundamental independent parameter. For each lattice mismatch, we then compare the ideal work of adhesion for a number of different boundary conditions. First, in the case of a semicoherent interface, both lattices are unstrained except for the local strain associated with the atomic relaxations that give rise to the formation of misfit dislocations. This is the physically realistic case (for all but the thinnest films).

The remaining types of boundary conditions all involve registry of the two subcrystals. While they imply artificial strain in one or both of the lattices, they are all easily implemented because they allow for the use of a unit cell whose dimensions in the interfacial plane can theoretically be as small as that of the primitive cell of the lattice. In the case of 'tensile' coherent boundary conditions, the lattice with the larger atoms is at its equilibrium lattice spacing while the other lattice is uniformly stretched so as to achieve perfect registry. 'Compressive' coherent boundary conditions are the exact analogue where the lattice with the smaller atoms is unstrained while the other lattice is compressed. Finally, we define coherent 'balanced' conditions that lie exactly halfway between the two extremes: one lattice gets suitably stretched and the other one equally compressed so as to achieve perfect registry.

Neglecting all effects related to either temperature or dynamics, our interest is in the ideal adhesive energy or work of adhesion $W_{\text {ad }}$. It is defined as the total potential energy of the separated lattices at infinite distance minus the total potential energy of the system when the two materials are joined at the interface. In other words, the work of adhesion is the thermodynamic work required to separate two materials at their interface, thus creating two new free surfaces. In the semicoherent case, both energies are obtained from energy 
minimization with respect to all degrees of freedom of the system. For coherent boundary conditions, on the other hand, the two lattices are internally held rigid and the energy is only minimized with respect to the interfacial distance parameter. This exactly mimics the approach employed in most quantum-mechanical determinations of the work of adhesion [3-12].

In the following, we first describe the precise setup of our model calculations for a Lennard-Jones bicrystal. We then present and discuss the influence of choice of boundary conditions on the work of adhesion.

\section{Methods}

The model system consists of two close-packed lattices, A and B, that differ only in the size and the number of atoms. In the $2 \mathrm{D}$ case, the interface is parallel to one of the closepacked directions in the close-packed triangular lattice. In the 3D case, both lattices are face-centred cubic and the interfaces are created by joining $\{111\}$ or $\{100\}$ surfaces with the same orientation with respect to the laboratory frame.

The particles interact with a Lennard-Jones potential, modified to give a smooth cutoff

$$
U\left(r_{i j}\right)=S\left(r_{i j}\right) \cdot 4 \varepsilon_{T(i, j)}\left[\left(\frac{\sigma_{T(i, j)}}{r_{i}}\right)^{12}-\left(\frac{\sigma_{T(i, j)}}{r_{j}}\right)^{6}\right]
$$

where $r_{i j}$ is the distance between atoms $i$ and $j$ and the subscript $T(i, j)$ indicates the pair of types (A or B) of the interacting atoms $i$ and $j . S(r)$ is a tapering function that gradually switches the interactions off between an inner radius $r_{1}$ and an outer radius $r_{2}$ :

$$
S(r)=3\left(\frac{r_{2}-r}{r_{2}-r_{1}}\right)^{2}-2\left(\frac{r_{2}-r}{r_{2}-r_{1}}\right)^{3}
$$

The Lennard-Jones strength parameters are $\varepsilon_{\mathrm{AA}}=\varepsilon_{\mathrm{BB}}=71.4 \mathrm{~kJ} \mathrm{~mol}^{-1}$ and $\varepsilon_{\mathrm{AB}}=$ $0.5 \varepsilon_{\mathrm{AA}}=0.5 \varepsilon_{\mathrm{BB}}$. If the atoms of both materials are of the same size, the length scale parameters are $\sigma_{\mathrm{AA}}=\sigma_{\mathrm{BB}}=\sigma_{\mathrm{AB}}=2.22 \AA$. With these numerical values for $\varepsilon$ and $\sigma$, the summation of the nearest neighbour interactions in the resultant $3 \mathrm{D}$ crystal yields the cohesive energy of $\mathrm{Ni}$ (although this does not imply any attempt to realistically model $\mathrm{Ni}$ ). Similarly, the factor of 0.5 for the relative strength of the interactions across the interface is arbitrary; the particular choice makes the 3D adhesion energies roughly comparable to the corresponding values for adhesion between transition metals as calculated with semiempirical many-body potentials [16]. The parameters $\varepsilon_{\mathrm{AA}}$ and $\varepsilon_{\mathrm{BB}}$ are set equal to one another for the sake of simplicity.

For dissimilar materials, let A be the one with the larger atomic size and B the one with the smaller. We then define the lattice misfit $f$ as

$$
f \equiv \frac{\sigma_{\mathrm{AA}}}{\sigma_{\mathrm{BB}}}-1
$$

and use this as the independent variable in our calculations. For a given misfit $f$, the parameters $\sigma_{\mathrm{AA}}$ and $\sigma_{\mathrm{BB}}$ deviate from the reference value of $\sigma=2.22 \AA$ such that the average number density of atoms in the interfacial plane remains constant; in a $2 \mathrm{D}$ system this implies that

$$
\frac{1}{\sigma_{\mathrm{AA}}}+\frac{1}{\sigma_{\mathrm{BB}}}=\text { constant }
$$


and in a 3D system

$$
\frac{1}{\sigma_{\mathrm{AA}}^{2}}+\frac{1}{\sigma_{\mathrm{BB}}^{2}}=\text { constant. }
$$

We arbitrarily assume that system A is the one where the particle size is increasing with growing misfit $f$ (implying a simultaneous decrease of the particle size in $\mathrm{B}$, as per equation (4)). The size parameter for the $\mathrm{A}-\mathrm{B}$ interactions is always $\sigma_{\mathrm{AB}}=0.5\left(\sigma_{\mathrm{AA}}+\sigma_{\mathrm{BB}}\right)$.

The inner radii $r_{1}$ used in the switching function for the A-A and $\mathrm{B}-\mathrm{B}$ interactions are the distances to the nearest neighbours in the corresponding unstrained lattices. The corresponding cutoff radii are $r_{2}=2.1 r_{1}$. The radii $r_{1}$ and $r_{2}$ for the A-B interactions are obtained by taking the algebraic mean of the corresponding A-A and $\mathrm{B}-\mathrm{B}$ values.

Periodic boundary conditions are imposed in the interfacial plane, with a fixed cell size. Perpendicular to the interfacial plane we employ slabs of 40 layers each (most 2D systems) or 18 layers each (most 3D systems). Only for the smallest misfits is the number of layers smaller: for $f=0.1 \%$ in $2 \mathrm{D}$, the number of layers in each lattice is 20 ; for $f=2.5 \%$ in $3 \mathrm{D}$, the number of layers is either 9 each (for $\{111\}$ interfaces) or 8 each (for $\{100\}$ interfaces). We always work with a simple Cartesian coordinate system. In the 3D case, the unit cell vectors are taken along the [100] and [010] directions; for a close-packed system, this implies that there must be an even number of particles along at least one of these directions.

We typically employ zero stress ('free') boundary conditions for the outer surfaces of the two slabs. For comparison, we also carry out calculations where the two outermost layers of each slab are frozen (zero strain boundary conditions), except for the one degree of freedom associated with the interfacial gap.

We explicitly consider four different interfacial boundary conditions: (i) semicoherent conditions where both lattices $\mathrm{A}$ and $\mathrm{B}$ are unstrained (i.e. at their $T=0$ lattice parameter). (Note that because of non-nearest neighbour interactions the densities are about $1 \%$ and $5 \%$ larger, for $2 \mathrm{D}$ and 3D, respectively, than the often used close-packing densities of a pure Lennard-Jones system where the nearest neighbour position is at the minimum of the pair potential.) We also employ (ii) tensile coherent boundary conditions where both lattices are brought into commensuration by straining material $\mathrm{B}$ (the one with the smaller atomic size), (iii) compressive coherent boundary conditions where material A is strained, and (iv) 'balanced' coherent boundary conditions where commensuration is achieved by straining both materials B and A exactly half as much as in the previous two cases.

Two ways of implementing a given strain are investigated. These are (i) biaxially straining one lattice parallel to the interface while keeping the total volume of the strained material constant, and (ii) biaxially straining one lattice parallel to the interface while keeping the lattice parameter in the orthogonal direction (z) fixed at its bulk value. We denote these two cases as 'constant volume' and 'constant $\Delta z$ ' conditions, respectively. For the former, the strain behaviour looks as if Poisson's ratio had a value of $v=1$ (2D) or $v=1 / 2$ (3D). For the latter, Poisson's ratio is formally $v=0$.

The energy minimizations are carried out in the following way. For the coherent interfaces, the atoms are fixed in their perfect crystal $(T=0)$ lattice positions and the energy is minimized with respect to the separation between the two crystals (i.e. the interfacial separation) using a deepest descent algorithm. For the semicoherent interface, the procedure varies slightly with the boundary conditions that are used for the outer surfaces of the two slabs of material. In the case of zero-stress boundary conditions (free outer layers), a PolakRibière conjugate gradient method [17] is used to relax the atomic positions of all particles, with the calculations deemed converged when the total energy varies by less than $10^{-5} \%$ 
in a single step. In the case of zero-strain boundary conditions (frozen outer layers), the steepest descent method (for the interfacial separation between rigid sublattices) and the conjugate gradient method (for the positions of all atoms other than those in the frozen outer layers) are used in alternating steps.

From the 'interfacial separation relaxed' energies, the ideal work of adhesion can be directly obtained as the energy associated with the A-B interactions at equilibrium and infinite separation. For the 'fully relaxed' systems, the work of adhesion has to be explicitly calculated as the difference of total energies, after carrying out energy minimizations with the two lattices first being in contact and then being at infinite separation. Note that the surface energies give rise to additional calculations; altogether 14 energy minimizations are required for the complete characterization of a system at a given misfit.

The misfit is varied between an upper limit of more than $30 \%$ and a lower limit of $0.1 \%$ and $2.5 \%$ in $2 \mathrm{D}$ and $3 \mathrm{D}$, respectively. The particular misfit values are chosen such that the misfit strain between the two lattices can be represented as a ratio of integer number of unit cells at the interface. The total number of atoms within the bicrystal lies between $\sim 800$ and $\sim 40000$ in the $2 \mathrm{D}$ case and between $\sim 4000$ and $\sim 59000$ in the 3D case.

\section{Results and discussion}

We are interested in the error that arises if the ideal work of adhesion is calculated with coherent boundary conditions, as is common in quantum-mechanical calculations, rather than with the semicoherent boundary conditions that are appropriate for all but very thin films. We first consider the results for simple 2D Lennard-Jones systems and then proceed to 3D systems, where we consider (111)\|(111) and (001)\|(001) interfaces in face-centred cubic lattices.

At most commonly encountered semicoherent bimaterial interfaces, the misfit dislocations reside at the interface. This is also how we set up our semicoherent interfaces. However, if the elastic properties of the two materials are very different, combinations of material parameters exist such that the dislocations may stand-off from the interface by a finite distance $[18,19]$ - a situation that is not dealt with here.

\section{Ideal work of adhesion, 2D systems}

In figure 1, the curve labelled 'semicoherent' gives the thermodynamic work of adhesion of our 2D model. This is the true zero temperature adhesion energy since the system is fully relaxed with respect to all degrees of freedom and the only stress present in the system is that due to the formation of misfit dislocations. Based on the fact that the dislocation spacing becomes smaller with increasing misfit, we expect the interfacial energy to increase and the work of adhesion to drop with increasing misfit. This is borne out by the data (figure 1). The rapid drop in the work of adhesion with increasing misfit for small misfit is attributable to the logarithmic variation of the energy of a dislocation with dislocation spacing (i.e. inverse misfit). In fact, a simple dislocation model shows that the interfacial energy rises infinitely fast with increasing misfit near zero misfit and saturates at large misfit [16].

The other three curves in figure 1 refer to the three types of coherent boundary conditions-'tensile', 'balanced', and 'compressive'-described earlier. The work of adhesion values of the three coherent interfaces all overestimate the true work of adhesion in the misfit range shown and exhibit a qualitatively different dependence on misfit than the work of adhesion of the semicoherent interface (especially at small misfit). This is not 


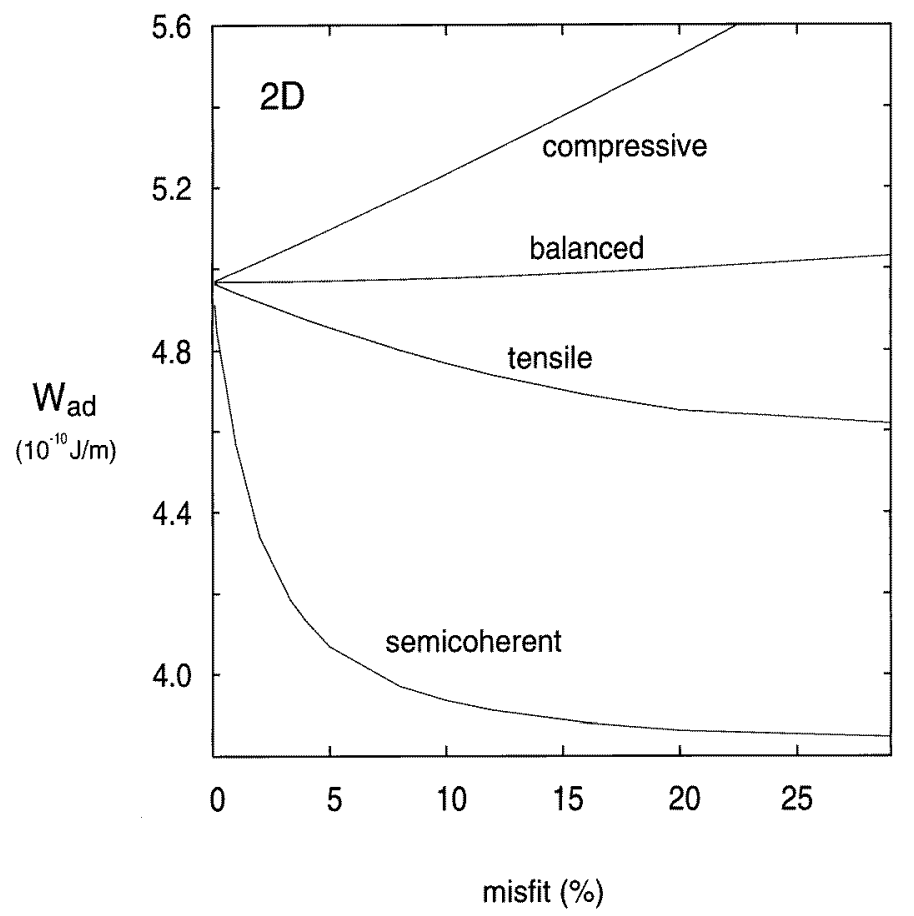

Figure 1. Work of adhesion between 2D Lennard-Jones solids, for the interfacial conditions as annotated (simulations were performed under 'constant volume' conditions). The small absolute values of the work of adhesion were obtained because of the 2D nature of these simulations (the values can be converted into effective 3D units by assuming that the material is one interatomic spacing wide-in which case typical works of adhesion are of order $1 \mathrm{~J} \mathrm{~m}^{-2}$ ).

surprising since the small misfit variation of the work of adhesion in the semicoherent case is dominated by elastic effects which are not present at all in the coherent calculations.

The corresponding errors (in \%) as a function of misfit, are shown in figure 2 (main panel). The smallest relative error is obtained with the tensile conditions, and the largest relative error with the compressive conditions. Even under the most favourable (tensile) conditions, the maximum error is $\sim 21 \%$, at a misfit of $\sim 10 \%$. Under balanced conditions, a maximum error of $\sim 37 \%$ is reached at a large misfit, $\sim 80 \%$ (not shown), while under compressive conditions the error is still monotonically increasing with increasing misfit even when the latter takes on very large and physically irrelevant values. Note that these errors are not associated with the strain energy stored within the strained lattice since the work of adhesion is calculated as the difference in energy of the system at the equilibrium interface separation and infinite interface separation at exactly the same strain.

The omission of misfit dislocations under coherent boundary conditions is expected to lead to an overestimate of the ideal work of adhesion and must, at least partially, explain the observed behaviour. The superiority of tensile coherent over compressive coherent conditions can also be rationalized if we correlate the ideal work of adhesion with the number of bonds per unit length (per unit area in 3D) across the interface and if we, moreover, neglect all structural relaxation effects (or, more precisely, all strain-induced changes of the structural relaxation). If we then consider how the number of bonds per unit length (per unit area in 3D) is affected by strain, tensile conditions should lead to 


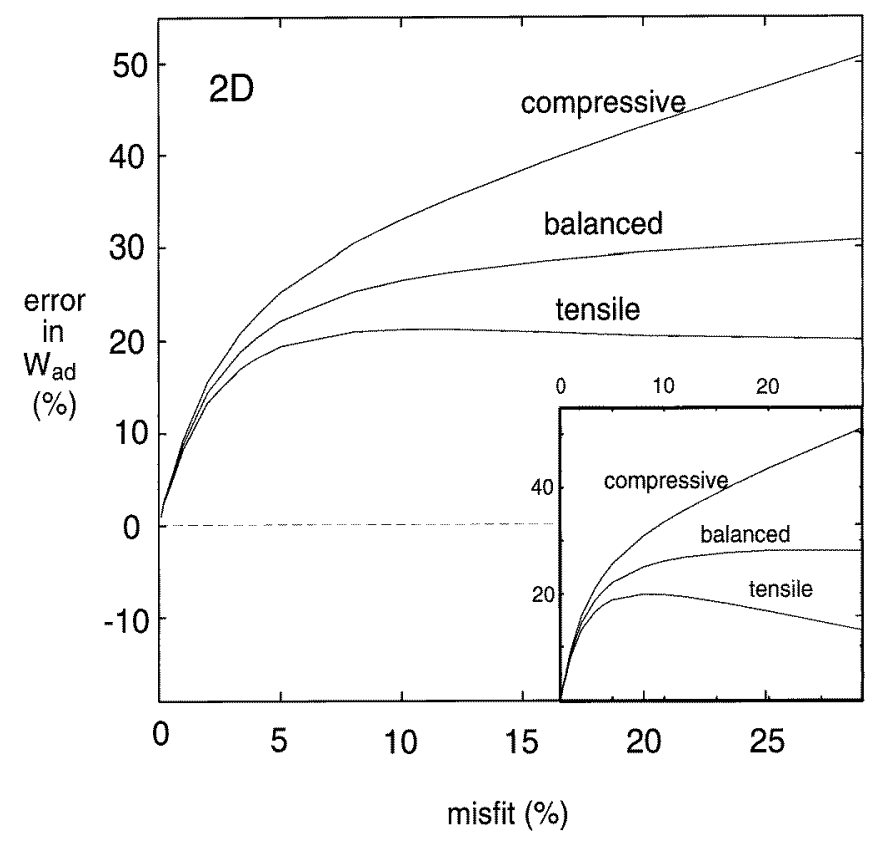

Figure 2. Relative error in the calculated ideal work of adhesion between 2D Lennard-Jones solids, for the annotated coherent boundary conditions and for semicoherent interface conditions. Main panel: Simulations performed with 'constant volume' constraints (corresponding to figure 1). Inset: Simulations performed with 'constant $\Delta z$ ' conditions.

a reduction in the number of bonds (since nearest neighbour bonds lower the energy this implies a larger interface energy and a smaller work of adhesion) and compressive conditions to a corresponding increase (smaller interface energy and larger work of adhesion). This simple description is consistent with the large misfit data. Superimposed on this effect is another which tends to increase the error (in a positive sense) with increasing misfit for all three coherent boundary conditions. This other effect appears to be dominant at small misfit and is still significant at large misfit (since the slope of the error with respect to misfit is not opposite for the tensile and compressive boundary conditions). It is associated with the elastic energy of the dislocations, which are present in the semicoherent case but not in the limit of coherency. At large misfit, there is some degree of error cancellation in the tensile case. On the other hand, the errors add in the compressive case.

The data shown in the main panel of figure 2 were obtained with 'constant volume' conditions where, for example, tensile strain is applied under simultaneous compression along the interface normal (i.e. we formally impose $v=1$ in 2D). The curves shown in the inset of the same figure were obtained with 'constant $\Delta z$ ' boundary conditions where the lattice spacing along the interface normal is kept at its equilibrium value (formally, $v=0$ ). While there are small quantitative differences between the constant volume and $\Delta z$ curves, they are in excellent qualitative agreement. There are no discernible differences between simulations performed with free surfaces on the edge of the lattice opposite the interface (i.e. zero-stress conditions) and those performed by fixing the atoms near the outer surface at their perfect crystal locations (i.e. zero-strain conditions). The results shown in figures 1 and 2, and in all subsequent figures, are for zero-stress boundary conditions on the surface opposite to the interface. 
Ideal work of adhesion, $3 D$ systems

In $3 \mathrm{D}$, the absolute values for the work of adhesion can be given physical significance. With a misfit of 5\%, for example, the ideal work of adhesion for our Lennard-Jones system (fit to the properties of $\mathrm{Ni}$ ) is $3.6 \mathrm{~J} \mathrm{~m}^{-2}$ for the (111)\|(111) interface and $3.1 \mathrm{~J} \mathrm{~m}^{-2}$ for the $(001) \|(001)$ interface. This is of the same order of magnitude as the adhesion energies that seem to be typical for interactions between transition metals [16].

The variation of the work of adhesion with misfit in 3D is qualitatively similar to that seen in 2D (and, hence, is not reported). Figures 3 and 4 show the errors in the calculated work of adhesion for the (111)\|(111) and (001)\|(001) interfaces, respectively, in exact analogy to figure 2. The qualitative behaviour of these curves is very similar to that seen in the $2 \mathrm{D}$ case; i.e. the coherent tensile strain case exhibits the smallest errors (relative to the appropriate semicoherent interface results) under both constant volume and constant $\Delta z$ conditions (main panels and insets, respectively). For adhesion between $\{111\}$ surfaces, the maximum error using coherent tensile strain $(\sim 13 \%)$ is somewhat smaller than in the 2D case $(\sim 21 \%)$, while the error is larger than in the 2D case at large misfit. For the (001)\|(001) interface, the error can be significantly larger (errors in excess of $\sim 50 \%$ for misfits of $\sim 10 \%$ ).

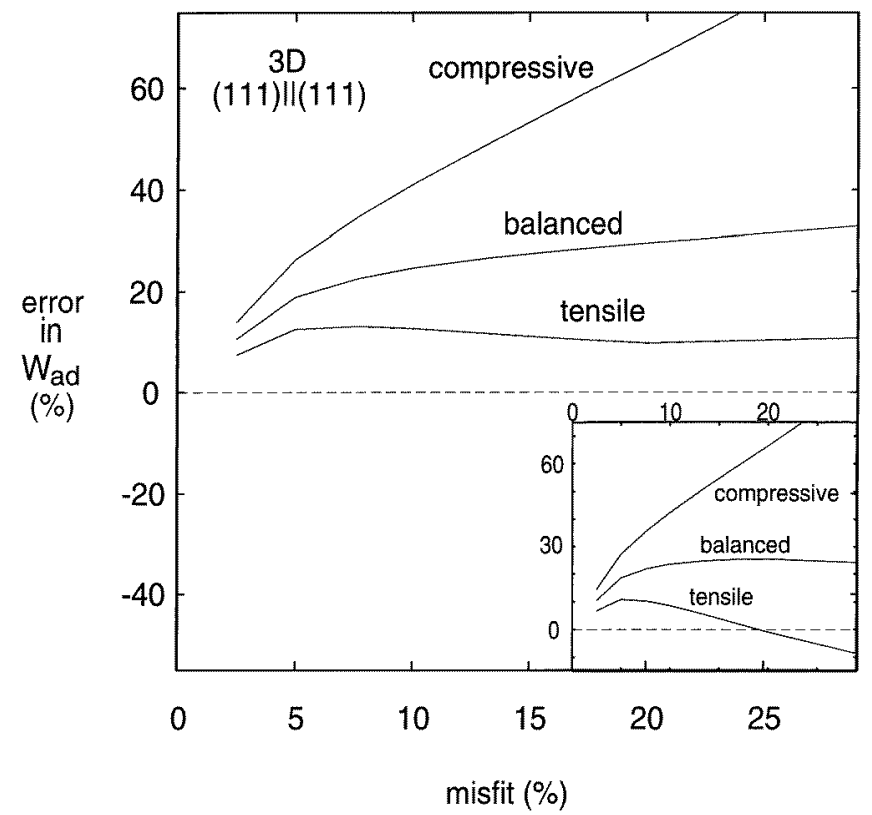

Figure 3. Relative error in the calculated ideal work of adhesion of the (111)\|(111) bimaterial interface in 3D Lennard-Jones solids, for the annotated coherent boundary conditions and for semicoherent interface conditions. Main panel: Simulations performed with 'constant volume' constraints (corresponding to figure 1). Inset: Simulations performed with 'constant $\Delta z$ ' conditions.

The inset in figure 3 shows that in some cases the ideal work of adhesion can be underestimated by a calculation based upon coherent boundary conditions (see the coherent tensile strain results under constant $\Delta z$ conditions for misfits that are in excess of $\sim 20 \%$ ). At the crossover point the true adhesion energy is exactly reproduced by the computationally much less expensive coherent tensile strain calculation, due to perfect cancellation of the 


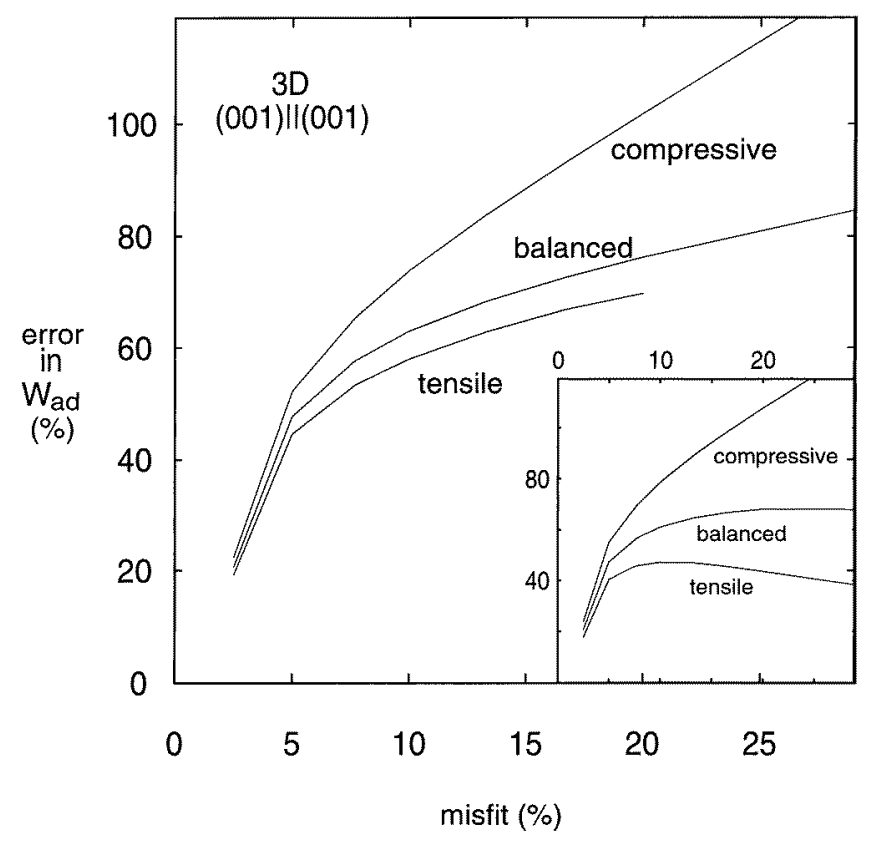

Figure 4. As per figure 3, but for the (001)\|(001) bimaterial interface between 3D Lennard-Jones solids.

errors that are associated with the omission of misfit dislocations and the change in the interfacial density. It is important to note, however, that this is a singular point and exact cancellation only occurs in exceptional situations.

\section{Surface energies and interfacial energy}

The ideal work of adhesion is the sum of the surface energies $\gamma_{\mathrm{A}}$ and $\gamma_{\mathrm{B}}$ of the two lattices minus the interfacial energy $\gamma_{\mathrm{AB}}$

$$
W_{\mathrm{ad}}(\mathrm{A}-\mathrm{B})=\gamma_{\mathrm{A}}+\gamma_{\mathrm{B}}-\gamma_{\mathrm{AB}} .
$$

The surface energies are simply half of the ideal work of adhesion of the corresponding pure materials:

$$
\begin{aligned}
& 1 / 2 W_{\text {ad }}(\mathrm{A}-\mathrm{A})=\gamma_{\mathrm{A}} \\
& 1 / 2 W_{\mathrm{ad}}(\mathrm{B}-\mathrm{B})=\gamma_{\mathrm{B}} .
\end{aligned}
$$

Figure 5 shows, for the 2D system, how the three contributions to the work of adhesion vary with the misfit. All three energies add up to the ideal work of adhesion shown in figure 1 and the latter, in turn, gives rise to errors (with respect to the semicoherent interface results) as shown in the main panel of figure 2 .

Focusing first on the semicoherent results in the upper panels of figure 5, we note that the surface energy $\gamma_{\mathrm{A}}$ drops and the surface energy $\gamma_{\mathrm{B}}$ increases with increasing misfit, while the sum of these two energies remains very nearly constant. Recalling that we choose the atomic sizes such that the sum of the surface densities remains constant when varying the misfit, it is clear that the variation of the surface energies with misfit simply reflects corresponding changes in the surface densities of both the A and B atom lattices. Although 


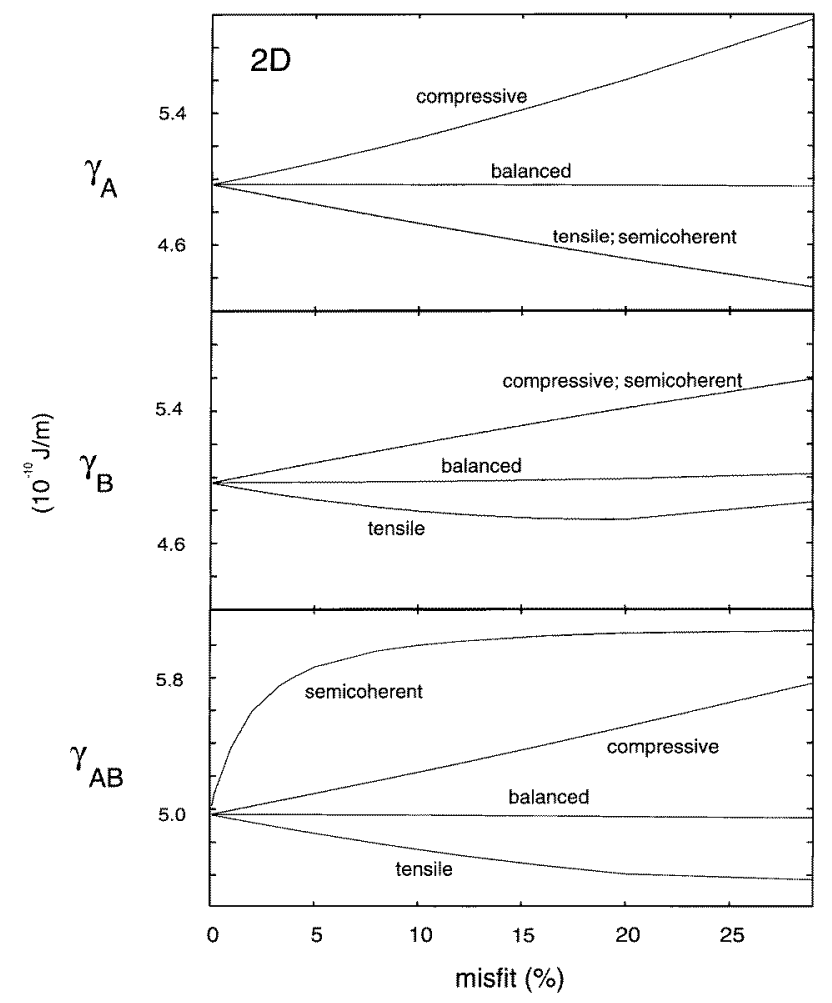

Figure 5. Surface and interfacial energy for $2 \mathrm{D}$ Lennard-Jones solids, using constant volume conditions. For each boundary condition, $\gamma_{\mathrm{A}}+\gamma_{\mathrm{B}}-\gamma_{\mathrm{AB}}$ is the ideal work of adhesion shown in figure 1.

the variation of the surface energies with misfit depends on the choice of atomic size, any choice is inherently arbitrary. Fortunately, these choices do not affect the relative ordering of the curves. The bottom panel of figure 5 indicates that the interfacial energy $\gamma_{\mathrm{AB}}$ does not go to zero in the vanishing misfit limit. This zero misfit interfacial energy is a direct measure of the unstretched chemical bond energy associated with the interface. In the present case, this value is positive since the $\mathrm{A}-\mathrm{B}$ bond strength is smaller than the A-A and $\mathrm{B}-\mathrm{B}$ interactions by a factor of two. Finally, we may attribute the sharp increase in the semicoherent interfacial energy $\gamma_{\mathrm{AB}}$ with increasing misfit near zero misfit with the large elastic energy required to introduce a dilute network of dislocations into an otherwise dislocation-free material (as occurs for a low angle grain boundary [20]).

Proceeding from semicoherent interfaces to the coherent tensile case, we note that the surface energy $\gamma_{B}$ of the strained component initially decreases with increasing misfit. This is because the number of $\mathrm{B}$ atoms per unit length on the surface (per unit area in 3D) decreases with misfit. On the other hand, the number of $A$ atoms per unit surface length (area) does not vary with misfit in this case. Based on this alone, the ideal work of adhesion should be expected to drop with increasing misfit. The interfacial energy $\gamma_{\mathrm{AB}}$, however, is also affected since the surface density of $B$ atoms also contributes to the number of atoms per unit length (area) on the interface. The interfacial energy $\gamma_{\mathrm{AB}}$ for coherent tensile conditions (bottom panel of figure 5) changes in such a way as to cause an increase in the ideal work of adhesion (recall that it is minus the interfacial energy that enters the work of adhesion). 
Since the work of adhesion in the coherent tensile case decreases with increasing misfit (figure 1), it is clear that the change of $\gamma_{\mathrm{AB}}$ is not large enough to overcome the decrease in the surface energy $\gamma_{\mathrm{B}}$. Therefore, the decrease in the coherent tensile work of adhesion with increasing misfit is largely attributable to the decrease in the number of bonds across the interface (relative to the unstrained or semicoherent case).

Under coherent compressive conditions, the number of A atoms per unit length (area) of the surface or interface increases (relative to the unstrained or semicoherent case). Therefore, the arguments made with respect to the coherent tensile case must be reversed when applied to the coherent compressive case since the number of atoms per unit length (area) increases here, while it decreases in the tensile case. While the surface energy $\gamma_{\mathrm{B}}$ is now the same as for the semicoherent case, the surface energy $\gamma_{\mathrm{A}}$ of the strained component increases with misfit. This increase is only partially offset by a corresponding decrease in the interfacial energy, still leading to the overall increase of the ideal work of adhesion (figure 1) that was earlier interpreted as an increase of the number of bonds across the interface.

Under balanced conditions, all simple surface and interfacial density effects have been eliminated. In particular, the interfacial energy $\gamma_{\mathrm{AB}}$ does not vary with misfit and the surface energies vary very slowly with misfit. The near constancy of each of the individual constituents of the coherent balanced work of adhesion is an indication that strain has only a minor effect on the surface and interfacial energies-provided that one properly normalizes the results with respect to the number of bonds.

Comparison of the surface and interfacial energy data presented in figure 5 with the work of adhesion data presented in figure 1 demonstrates that the work of adhesion is dominated by the surface energy and that the interfacial energy plays a relatively minor role. On the other hand, the variation of the work of adhesion with misfit in the experimentally relevant semicoherent case, is dominated by the dislocation energy. In the coherent interface case (pertinent to most first-principles calculations), the variation of the work of adhesion with misfit is dominated by the change in the number of bonds per unit length (area), except in the coherent balanced case.

\section{Conclusions}

We have presented clear evidence that the variation in the work of adhesion of semicoherent interfaces with misfit is dominated by the energetics of the dislocation network. On the other hand, the variation of the work of adhesion for coherent interfaces is dominated by the changes in the number of bonds per unit area. The common practice in the application of quantum-mechanically based methods to determine the thermodynamic work of adhesion of heterophase interfaces is to strain one or both materials into coherency. This approach can lead to errors of several tens of percent as compared with the typical experimentally relevant semicoherent case, unless the dislocation network is separately and explicitly accounted for. These errors increase very rapidly with increasing misfit at small misfit and more slowly at large misfit. The errors, while still large, are generally reduced by concentrating the misfit strain into a tensile strain in one lattice while leaving the second lattice unstrained. This is, however, a rather uncontrolled approach to minimizing the errors.

The use of coherent boundary conditions nevertheless continues to be of great appeal because they allow the use of unit cells small enough for computationally expensive quantum-mechanical calculations. Clearly, the ideal solution to computationally determine the work of adhesion of bimaterial interfaces is to use unstrained lattices and to minimize the total energy of the system with respect to the atomic coordinates in order to properly account for the core and elastic energies of the interfacial dislocations. While faster 
quantum-mechanical methods [21] that may allow for such calculations in relatively simple materials are currently under development, this approach remains relatively far off for most applications.

At the present time, the best approach to minimizing the error in quantum-mechanical calculations of the work of adhesion of bimaterial interfaces in systems with relatively low misfit (i.e. the ones most likely to be semicoherent, as opposed to incoherent), is to perform small, coherent interface calculations using balanced boundary conditions and to correct the results using analytical dislocation theory and an estimate of the dislocation core energies. This approach was alluded to in two recent studies [10,22]. Elasticity solutions are available for regular arrays of misfit dislocations at bimaterial interfaces [23] from which the elastic energy may be determined. Several methods for estimating the core energy are also available [20].

\section{Acknowledgments}

The authors would like to thank Dr John R Smith of General Motors Research and Development Center and Dr James Raynolds of Knowles Atomic Power Laboratory for useful discussions. Further, the authors gratefully acknowledge the Division of Materials Science of the Office of Basic Energy Sciences of the United States Department of Energy, Grant No FG02-88ER45367, for its support.

\section{References}

[1] Pantano C G and Chen E J H 1989 Interfaces in Composite Materials (Pittsburgh: Materials Research Society)

[2] 1990 Metal-Ceramics Interfaces ed M Rühle et al (Oxford: Pergamon)

[3] Freeman A J, Li C and Fu C L 1990 Metal-Ceramics Interfaces ed M Rühle et al (Oxford: Pergamon)

[4] Blöchl P, Das G P, Fischmeister H F and Schönberger U 1990 Metal-Ceramics Interfaces ed M Rühle et al (Oxford: Pergamon)

[5] Lambrecht W R L and Segall B 1990 Metal-Ceramics Interfaces ed M Rühle et al (Oxford: Pergamon)

[6] Eberhart M E, Crampin S, MacLaren J M and McHenry M E 1990 Metal-Ceramics Interfaces ed M Rühle et al (Oxford: Pergamon)

[7] Schönberger U, Andersen O K and Methfessel M 1992 Acta Metall. Mater. 40 S1

[8] Duffy D M, Harding J H and Stoneham A M 1992 Acta Metall. Mater. 40 S11

[9] Hong T, Smith J R and Srolovitz D J 1993 Interface Sci. 1223

Hong T, Smith J R and Srolovitz D J 1993 Phys. Rev. B 4713615

[10] Hong T, Smith J R and Srolovitz D J 1995 Acta Metall. Mater. 432721

[11] Zhao G-L, Smith J R, Raynolds J E and Srolovitz D J 1996 Interface Sci. 3289

[12] Raynolds J E, Smith J R, Zhao G-L and Srolovitz D J 1996 Phys. Rev. B 5313883

[13] Sutton A P and Balluffi R W 1995 Interfaces in Crystalline Materials (Oxford: Clarendon)

[14] Nandedkar A S, Srinavasan G R and Murthy C S 1991 Phys. Rev. B 437308 Nandedkar A S 1993 Acta Metall. Mater. 413455

[15] Quesnel D J, Rimai D S and DeMejo L P 1995 J. Adhesion Sci. Technol. 91015

[16] Schnitker J and Srolovitz D J to be published

[17] Press W H, Teukolsky S A, Vetterling W T and Flannery B P 1992 Numerical Recipes in C 2nd edn (Cambridge: Cambridge University Press)

[18] Mader W and Knauss D 1992 Acta Metall. Mater. 40 S207

[19] Shilkrot L E and Srolovitz D J Acta Mater. at press

[20] Hirth J P and Lothe J 1982 Theory of Dislocations 2nd edn (New York: Wiley)

[21] Galli G 1996 Current Opinion Solid State Mater. Sci. 1864

[22] Trampert A, Ernst F, Flynn C P, Fischmeister H F and Rühle M 1992 Acta Metall. Mater. 40 S227

[23] Hirth J P, Barnett D M and Lothe J 1979 Phil. Mag. A 4039 